\title{
Plantar fascia rupture in elite athlete of volleyball -
}

\section{case report}

\begin{abstract}
The literature explains its pathophysiology through a process where mechanical overload and excessive stress produce intrasubstantial microruptures in the plantar fascia. In high-performance athletes (elite), involved in running and jumping, repeated daily heel trauma without the recommended interval may hinder normal healing, can resulting in a rupture the plantar fascia. The plantar fascia rupture resulting from chronic local inflammation or previous infiltration with corticosteroids may occur spontaneously during elite athletic activity. The objective of this case report is to document the diagnosis, treatment proposal and time of return to the sport activity of Olympic athletes and volleyball professionals, whilst in the National League matches, after a start, reported audible click and sudden pain in right foot plantar region with impossibility of movement and march. It evolved with acute plantar fascia rupture without history of previous infiltrations of corticosteroids, entity rarely described in literature.
\end{abstract}

Volume I Issue 3 - 2017

\author{
Alexandre de Paiva Luciano, ${ }^{1,2}$ Thiago \\ Fukugauti, ${ }^{2}$ Miguel Ambrosio Neto ${ }^{2}$ \\ 'Department of Orthopedic Surgery, Taubate Medical School, \\ Brazil \\ ${ }^{2}$ Department of Medical and Phisiological-Funvic/Taubate \\ Volleyball Team, Brazil
}

Correspondence: Alexandre de Paiva Luciano, Department of Orthopedic Surgery, Taubate Medical School,Adress Tiradentes Avenue, 500 - Campus Bom Conselho - Zip Code I2030-180, Brazil,Email paivaortopedia@gmail.com

Received: May 26, 2017 | Published: July 05, 2017

Keywords: sports injuries, fascist, plantar, rupture

\section{Introduction}

Plantar fasciitis is the most common cause of pain in the calcaneus, near the origin in the median tuberosity. It is estimated that $11 \%$ to $15 \%$ of all foot complaints requiring medical attention can be attributed to this condition. ${ }^{1,2}$ The literature explains its pathophysiology through a process where mechanical overload and excessive stress produce intrasubstantial microruptures in the plantar fascia, which leads to an inflammatory response. ${ }^{3}$ In high-performance athletes (elite), involved in running and jumping, repeated daily heel trauma without the recommended interval may hinder normal healing, resulting in chronic inflammation followed by degeneration. ${ }^{4}$ Plantar fascia rupture resulting from chronic local inflammation or previous corticosteroid infiltration may occur spontaneously during elite athletic activity.,6 Therefore, the purpose of this case report is to document the diagnosis, propose a treatment and time of return to sports activity after acute plantar fascia rupture in a high-performance (elite) athlete without previous corticosteroid infiltrations, an entity rarely described in the literature. ${ }^{6,7}$

\section{Case description}

23-year-old patient; male, Olympic athlete and volleyball professional, began medical and physiotherapeutic follow-up at the club due to mild pain in the right calcaneus, mainly concentrated in the medial region of the hindfoot during the jumps and starts for 21 days, in physical therapy treatment with electroanalgesia and laser, without history of chronic use of infiltrations of corticosteroids, previous surgeries or chronic diseases previously diagnosed in the studied segment. Patient works in club and national team, with cumulative and daily training load of up to 6 hours per day for 12 months without intervals. In a match by National Volleyball League in Brazil after a start, patient mentioned audible click and sudden pain in the right foot plantar region with impossibility of movement and back to the march. We started treatment on the block with cryotherapy and immobilization with robofoot without load.

\section{Physical exam}

Physical examination of the right foot after 24 hours of injury (Figure 1)

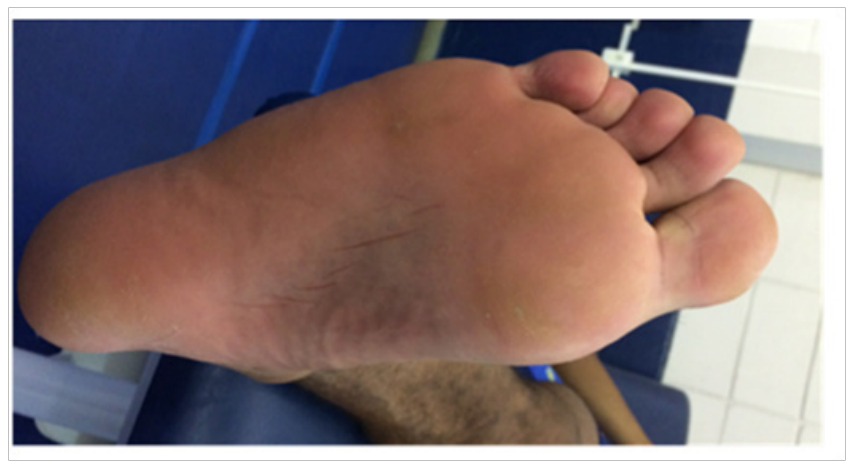

Figure I right foot plantar region with emphasis in medial calcaneus face.

i. Inspection: claudicating gait; without atrophies, retractions or bruises.

ii. Palpation: intense pain to the palpation of soft parts and of the plantar fascia, mainly in the medial region of the hind foot near the calcaneus.

iii. Degree of mobility: extension, flexion, lateral rotation, medial rotation, abduction, adduction.

iv. Neurological strength test of the ankle Grade V: complete movement against gravity and against great resistance.

At the same time, imaging examinations were requested: Magnetic Resonance (MRI) of the foot 24 hours after the injury on the court 03/03/2017 Figure 2, 3 showing signs of acute traumatic rupture of the plantar fascia at calcaneus origin, with small plantar net collection without significant shrinkage. Central plantar fasciitis is associated 
with fusiform thickening of the fibers and degeneration area near the origin of the calcaneus, close to the area of traumatic rupture.
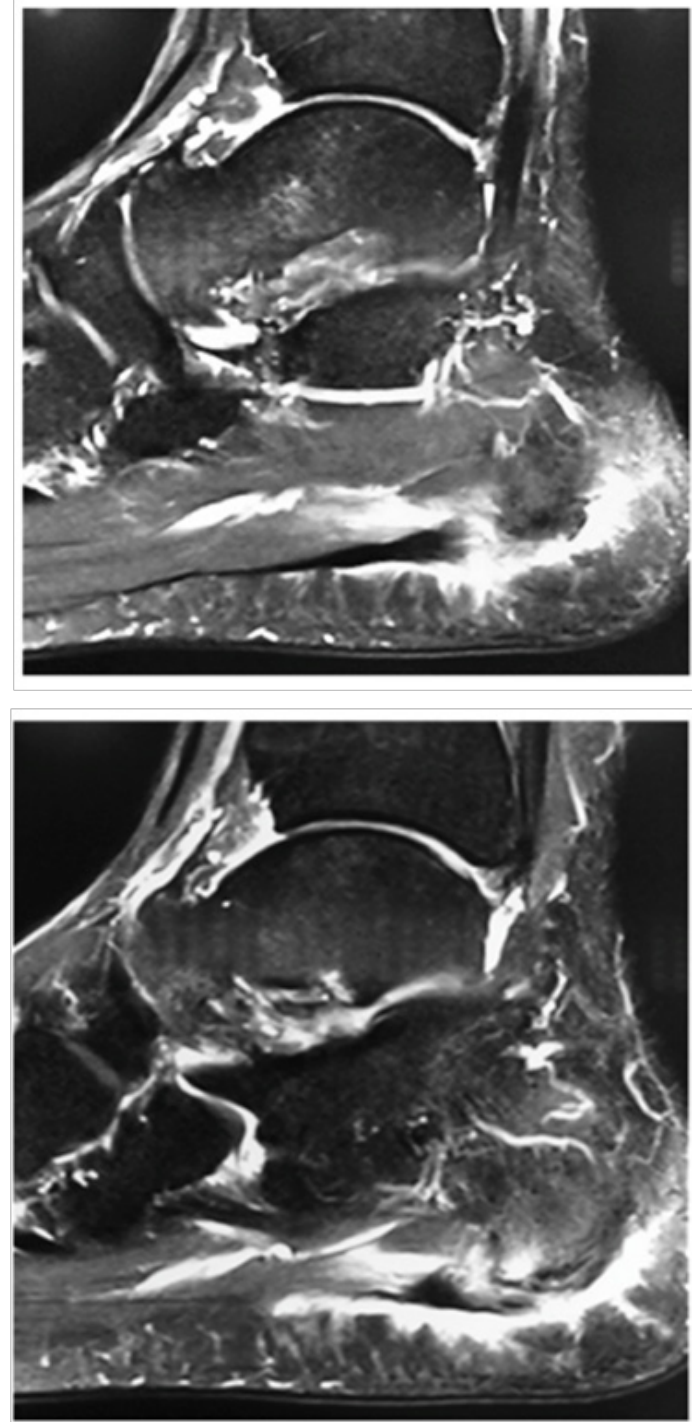

Figure 2, 3 Magnetic Resonance of the foot performed with multiplanar cuts of $4.0 \mathrm{~mm}$ showing signs of acute traumatic rupture of the plantar fascia at the calcaneus origin.

\section{Treatment}

After diagnostic confirmation, non-hormonal anti-inflammatory lesion (NSAID) treatment was started for 7 days for pain relief and edema, immobilization with a 10-day unloaded orthopedic orthosis, and concomitant physiotherapy rehabilitation in 2 periods for 5 weeks in the following protocol

\section{I $^{\text {st }}$ and $2^{\text {nd }}$ Physiotherapeutic rehabilitation weeks}

i. Laser: 4 Joules - 20 points + Led 9 Joules;

ii. Flexibility training of the following muscle groups: adductor, hamstrings, fascia lata tensor, buttocks, quadriceps, ventral decubitus spine extension.

iii. Electroanalgesia with interference to improve tissue healing.

\section{$3^{\text {rd }}$ Physiotherapeutic rehabilitation week}

i. Laser: 6 Joules ( 8 points) + Led 6 Joules ( 8 points);

ii. Russian current for maintenance of gastrocnemius muscle tone (15 minutes, 40 seconds ON, 20 seconds OFF)

iii. Pool training for 50 minutes: with lateral displacement heating + front back (functional blocking /attack), attack coupling, contact with the ball (touch) + lateral displacement, contact with the bole (touch) + displacement front back.

\section{$4^{\text {th }}$ Physiotherapeutic rehabilitation week}

i. Return to group training according to protocol: attack/defense training (no recovery of ball requiring a run), training of pass (except short throw), total restriction to jumps and Laser 6 Joules ( 8 points) + Led 6 Joules ( 8 points)

\section{$5^{\text {th }}$ Physiotherapeutic rehabilitation week}

After the 5th week of rehabilitation without jumping restrictions, magnetic resonance (MR) control was requested, showing a thickening of the plantar fascia next to its calcaneal insertion with a maximum thickness of $9 \mathrm{~mm}$ showing increased signal intensity with healing of its fibers in its portion More medial (Figure 4) (Figure 5). There is evidence of plantar fascia thickening next to its calcaneal insertion with $9 \mathrm{~mm}$ of thickness. After confirming the healing process, the athlete was available to the technical committee for training and games at the end of the 5 th rehabilitation week.
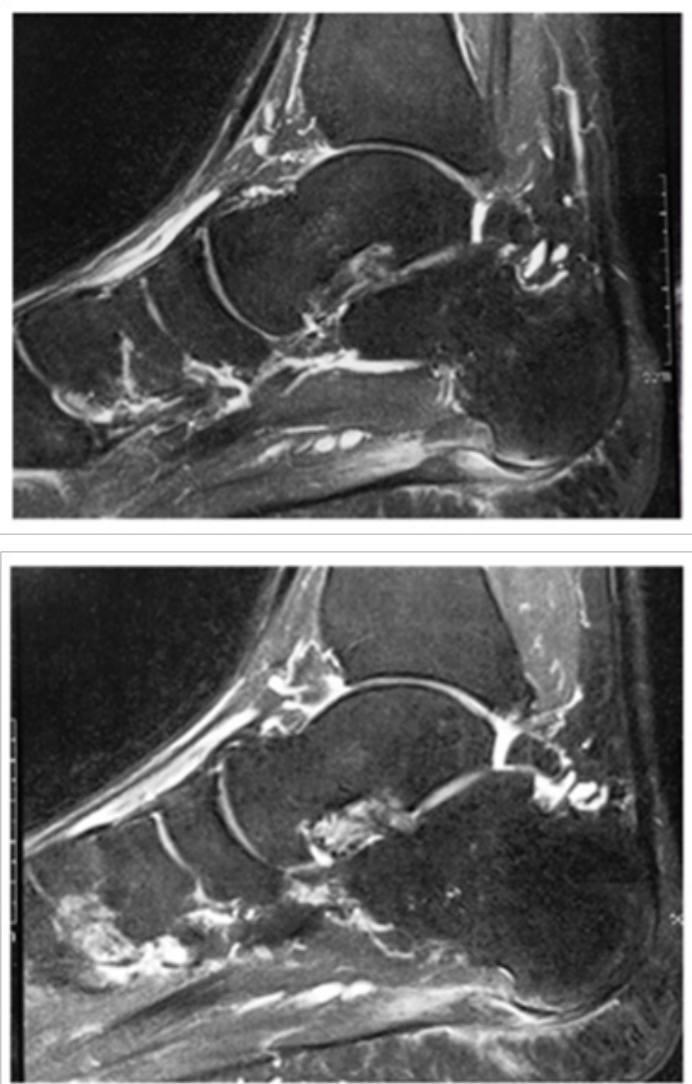

Figure 4, 5 Magnetic resonance control with T2-weighted multiplanar FAT SAT sequences. 


\section{Discussion}

Systematic reviews of the literature indicate that knee injuries are among the most common in volleyball and represent $33 \%$ of acute injuries, followed by ankle (17\%) and shoulder (17\%). For overuse injuries, knee and shoulder injuries that represent, respectively, 24\% and $12 \%$ of overuse injuries in volleyball have been reported ${ }^{8}$

Plantar fascia ruptures may represent an acute lesion on chronic plantar fasciitis on previous treatment with corticosteroid injections, leading to weakening of the plantar fascia and may evolve with future acute rupture. Leach et al. ${ }^{7}$ reported that in 6 ruptures of the plantar fascia, 5 had previously been injected. Sellman ${ }^{9}$ reported 37 patients with ruptures, all associated with prior corticosteroid injection. Acevedo et al. ${ }^{5}$ reported 51 ruptures, with 44 cases that underwent injection therapy. The athlete in question at no point in his career was treated with corticosteroid injections for plantar fasciitis and the mechanism of acute injury of the plantar fascia was due to the initiation movement with excessive overload in the plantar fascia which attests the rarity of the presented case. Since there is limited evidence about the value of physical therapy treatments for plantar rupture, the recommendations generally reflect current clinical practice, but most of them are of unproven benefit. ${ }^{6,7}$ Therefore, the treatment protocol presented in this study is also based on the clinical practice of the specialists and becomes an option to increase knowledge about this rare lesion. In the literature consulted, the treatment of acute pain included the use of crutches during pain, cryotherapy for 8 to 15 days and phenylbutazone $100 \mathrm{mg} 3$ times a day for 6 to 8 days with foot protection with insoles and gradual body load after 2 a 3 weeks post - injury. ${ }^{8}$

In the athlete reported, we started loading with crutches after 10 days of the trauma and he was allowed to walk without crutches support 15 days post - injury, even without pain symptoms at the beginning of gait. Magnetic resonance imaging (MRI) is valued in the literature and should assess severity and degree of rupture. ${ }^{8}$ In the case in question, we used MRI for the initial diagnosis and return of the athlete to the court. However, we did not find MRI indication at the end of the treatment in the researched literature.

The athlete in question returned to unrestricted practice at the end of the 5th week, which is in agreement with the consulted literature recommending a period of approximately 9 weeks for total reintegration into the sport. ${ }^{8}$ In this case the athlete evolved without residual pain and/or sequelae in relation to the longitudinal arch of the injured foot, suggesting the successful treatment described. Therefore, there is an agreement in the research on the use of a south-pedal orthosis, a period without body load, physiotherapy and adequate use of foot orthoses leading to complete recovery of plantar fascia rupture. ${ }^{5,8,9,11}$ However, there is no consensus of a physiotherapeutic protocol for the treatment of this lesion. The efficacy of preventive strategies such as stretching exercises and control of the intensity of running (e.g., limiting distance, frequency, and duration) is not known. ${ }^{12}$ We conclude that more studies are needed to evaluate the physiotherapeutic protocol indicated in the acute lesions of the plantar fascia, to measure the importance of MRI evaluation not only to assess severity and degree of rupture, but as a criterion of backward sportive activity.

\section{Acknowledgements}

None.

\section{Conflict of interest}

No potential conflict of interest was reported by the authors.

\section{References}

1. League AC. Current concepts review: plantar fasciitis. Foot Ankle Int 2008;29(3):358-366.

2. McCarthy DJ, Gorecki GE. The anatomical basis of inferior calcaneal lesions: a cryomicrotomy study. J Am Podiatry Assoc. 1979;69(9):527536.

3. Kaya BK. Plantar fasciitis in athletes. J Sport Rehabil. 1996;5(4):305320.

4. LeMelle DP, Kisilewicz P, Janis LR. Chronic plantar fascial inflammation and fibrosis. Clin Podiatr Med Surg. 1990;7(2):385-389.

5. Acevedo JI, Beskin JL. Complications of plantar fascia rupture associated with corticosteroid injection. Foot Ankle Int. 1998;19(2):91-97.

6. Buchbinder R. Plantar fasciitis. New England Journal of Medicine. 2004;350(21):2159-2166.

7. Janmes LT, steven RK, Robert WM, et al. The diagnosis and treatment of heel pain. J Foot Ankle Surg. 2001;40(5):329-340.

8. Saxena A, Fullem B. Plantar fascia ruptures in athletes. The American Journal of sports medicine. 2004;32(3):662-665.

9. Kilic, M Maas, E Verhagen, et al. Incidence, etiology and prevention of musculoskeletal injuries in volleyball: A systematic review of the literature. Eur J Sport Sci. 2017;17(6):765-793.

10. R Leach, R Jones, T Silva. Rupture of the plantar fascia in athletes. $J$ Bone Joint Surg Am Jun. 1978;60(4):537-539.

11. Sellman JR. Plantar fascia rupture associated with corticosteroid injection. Foot Ankle Int. 1994;15(7):376-381.

12. Yeung EW, Yeung SS. A systematic review of interventions to prevent lower limb soft tissue running injuries. Br J Sports Med. 2001;35(6):383389. 